\title{
Malnutrition risk at solid tumor diagnosis: the malnutrition screening tool in a large US cancer institute
}

\author{
Kunal C. Kadakia ${ }^{1,2}\left(\right.$ D . James T. Symanowski ${ }^{3} \cdot$ Aynur Aktas $^{2} \cdot$ Michele L. Szafranski $^{2}$. Jonathan C. Salo ${ }^{4}$. \\ Patrick L. Meadors ${ }^{2}$. Declan Walsh ${ }^{2}$
}

Received: 19 May 2021 / Accepted: 30 September 2021 / Published online: 28 October 2021

(c) The Author(s), under exclusive licence to Springer-Verlag GmbH Germany, part of Springer Nature 2021

\begin{abstract}
Background In cancer, malnutrition is common and negatively impacts tolerance and outcomes of anti-tumor therapies. The aim of this study was to evaluate the prevalence of malnutrition risk and compare the clinicodemographic features between those with high malnutrition screening tool (MST) scores (i.e., $\geq 2$ of $5=$ high risk for malnutrition, H-MST) to low scores (L-MST).

Methods A cohort of 3585 patients (May 2017 through December 2018), who completed the MST at least once at the time of diagnosis of any stage solid tumor, were analyzed. Logistic regression tested for associations between clinicodemographic factors, symptom scores, and H-MST prevalence.

Results The median age was 64 years (25-75 IQR, 55-72), with $62 \%$ females and $81 \%$ White. Most common tumor primary sites were breast (28\%), gastrointestinal (GI) (21\%), and thoracic (13\%). Most had non-metastatic disease (80\%). H-MST was found in 28\% - most commonly in upper (58\%) and lower GI (42\%), and thoracic (42\%) tumors. L-MST was most common in breast (90\%). Multivariable regression confirmed that Black race (OR 1.9, 95\% CI 1.5-2.4, $p=<0.001$ ), cancer primary site (OR 1.6-5.7, $p=<0.001$ ), stage IV disease (OR 1.8, 95\% CI 1.4-2.2, $p=<0.001$ ), low BMI (OR 4.2, 95\% CI 2.5-6.9 $p=<0.001)$, and higher symptom scores were all independently associated with H-MST.

Conclusions Twenty-eight percent of solid tumor oncology patients at diagnosis were at high risk of malnutrition. Patients with breast cancer rarely had malnutrition risk at diagnosis. Significant variation was found in malnutrition risk by cancer site, stage, race, and presence of depression, distress, fatigue, and trouble eating/swallowing.
\end{abstract}

Keywords /MeSH: Malnutrition $\cdot$ Cancer $\cdot$ Screening $\cdot$ Nutrition assessment $\cdot$ Medical oncology

Kunal C. Kadakia

Kunal.Kadakia@atriumhealth.org

James T. Symanowski

james.symanowski@atriumhealth.org

Aynur Aktas

aynur.aktas@atriumhealth.org

Michele L. Szafranski

michele.szafranski@atriumhealth.org

Jonathan C. Salo

jonathan.salo@atriumhealth.org

Patrick L. Meadors

patrick.meadors@atriumhealth.org
Declan Walsh

declan.walsh@atriumhealth.org

1 Department of Solid Tumor Oncology and Supportive Oncology, Levine Cancer Institute, Atrium Health, 1021 Morehead Medical Drive, Charlotte, NC 28204, USA

2 Department of Supportive Oncology, Levine Cancer Institute, Atrium Health, Charlotte, NC, USA

3 Department of Cancer Biostatistics, Levine Cancer Institute, Charlotte, NC, USA

4 Department of Surgery, Division of Surgical Oncology, Levine Cancer Institute, Charlotte, NC, USA 


\section{Introduction}

In cancer, multiple factors contribute to malnutrition including tumor-related symptoms (e.g., anorexia, early satiety, fatigue), treatment-related complications (e.g., dysgeusia, mucositis, nausea), and psychological distress. [1] The prevalence of cancer-related malnutrition ranges from 30 to $80 \%$ in ambulatory and hospitalized patients. [2-7] Malnutrition has profound negative effects on performance status (PS), psychological well-being, and overall quality of life (QoL). [8-10] Importantly, malnutrition is a predictor of cancer survival independent of cancer site, PS, or stage. [11] Despite this, routine screening for malnutrition in oncology is the exception with underutilization of potentially helpful nutritional support services. [12-15]

Guidelines from the European Society for Clinical Nutrition and Metabolism (ESPEN) support universal nutritional screening as it might allow early nutritional interventions. [16] Multiple validated malnutrition screening tools of varying structure are available and incorporate patient, clinician, and objective data. [17, 18] Developed in 1999, the malnutrition screening tool (MST) is a short tool validated in oncology outpatients (Table 1). [3, 19, 20] An observational, cross-sectional study of 50 chemotherapy patients determined relative MST validity compared to the PatientGenerated Subjective Global Assessment (PG-SGA). MST was a strong predictor of malnutrition risk relative to the PG-SGA (100\% sensitivity, 92\% specificity, 0.8 positive predictive value, 1.0 negative predictive value). [21] Due to

Table 1 Malnutrition screening tool

\begin{tabular}{ll}
\hline Question & Score \\
\hline Have you lost weight recently without trying? & 0 \\
No & 2 \\
Unsure & See below \\
Yes & \\
If yes, how much weight $(\mathrm{kg})$ have you lost? & 1 \\
$1-5$ & 2 \\
$6-10$ & 3 \\
$11-15$ & 4 \\
$>15$ & 2 \\
Unsure & 0 \\
Have you been eating poorly because of a decreased appetite? & \\
No & 1 \\
Yes & \\
Total* & \\
\hline
\end{tabular}

* Subjects who score 2 to 5 are at risk for malnutrition. Reprinted from Nutrition, Volume 15, Issue 6, "Development of a valid and reliable malnutrition screening tool for adult acute hospital patients," Ferguson M et al. Pages 458-464, Copyright 1999, with permission from Elsevier ${ }^{20}$ its ease of administration and test characteristics, the MST can screen cancer patients with relatively high sensitivity and specificity.

In January 2017, the Levine Cancer Institute (LCI), a high-volume, not-for-profit tertiary care academic-community cancer center, initiated an electronic distress screening (EDS) process. This aimed to screen all ambulatory oncology patients via an electronic tablet at initial consultation. The EDS includes patient-reported demographic, physical, and psychosocial parameters and linked to the electronic medical record. The MST was added to the EDS in May 2017 to identify those at high risk for malnutrition.

As the largest studies of malnutrition screening in oncology are from non-US populations, it is imperative to understand the prevalence of patient-reported malnutrition and associated clinicodemographic and physical/psychologic characteristics in a large US cohort. [7, 11, 22] This retrospective study analyzed a patient cohort with any solid tumor who completed the MST at diagnosis. Specifically, we compared the clinicodemographic features of those at high risk for malnutrition to those with low risk and identified features associated with high risk.

\section{Methods}

\section{Design}

A retrospective review was conducted of adult patients $\geq 18$ years of age diagnosed with any stage solid tumors and who completed at least one MST between May 2017 and December 2018. The Institution Review Board for Atrium Health approved this study, and requirements for consent were waived. The Strengthening the Reporting of Observational Studies in Epidemiology (STROBE) statement was used as a guide for this manuscript. [23]

\section{Setting}

LCI is a high-volume, not-for-profit tertiary care academiccommunity "hybrid" cancer center with a catchment area across 25 care locations in North and South Carolina.

\section{Data sources and variables}

\section{Data sources}

The LCI EDS database registry, the enterprise data warehouse, and the institutional tumor registry identified patients and variables. 


\section{Variables}

Cancer type and stage at diagnosis were obtained from the institutional tumor registry. Cancer diagnostic groups were established which included breast, genitourinary (GU), gynecologic $(\mathrm{GYN})$, head and neck $(\mathrm{H} \& \mathrm{~N})$, lower gastrointestinal (GI), other, thoracic, and upper GI. Sociodemographic variables included age at diagnosis, race, and sex. Patient characteristics included alcohol or tobacco use, height $(\mathrm{cm}$.$) , and weight (lbs.) (used to derive BMI [\mathrm{kg} /$ $\mathrm{m}^{2}$ ]) [24].

The EDS included the MST and patient-reported symptoms (anxiety, constipation/diarrhea, depression, distress, fatigued or tired, nausea/vomiting, pain, and trouble eating/ swallowing). MST scores range from 0 to 5 with scores $\geq 2$ indicating high risk for malnutrition (H-MST) and 0-1 low risk for malnutrition (L-MST). [21] Anxiety was measured using the two-item Generalized Anxiety Disorder (GAD-2) score range 0-6. [25] GAD-2 scores of $\geq 3$ are associated with high sensitivity and specificity for generalized anxiety disorder. Depression was assessed using the validated twoitem Patient Health Questionnaire (PHQ-2) score range 0-6. [26] PHQ-2 scores of $\geq 3$ are associated with high sensitivity and specificity for major depression; it has been validated in oncology. [27] Distress was based on a single item "How much distress have you been feeling in the past week?," low versus high, $\geq 4$ on $0-10$ scale. A single item distress score is supported by the National Comprehensive Cancer Network (NCCN) Distress Management Panel. [28] All other symptoms were measured for severity on a $0-10$ scale, a higher number reflecting greater symptom severity.

\section{Data processing}

The above data sources were merged to identify all unique patients with complete case records i.e., no missing variables. This included at least one complete EDS plus that MST done closest to the date of cancer diagnosis.

\section{Statistical methods}

MST scores were summarized descriptively overall and by cancer diagnostic groups separately. The prevalence of MST scores indicating high risk of malnutrition were modeled using univariate and multivariable logistic regression. Covariates included demographic variables (age, BMI, race, sex), oncologic variables (site and stage of cancer), and symptom scores. Patient-reported symptoms evaluated included anxiety and depression, distress score, and the following specific symptoms (as continuous variables on a 0-10 scale; constipation/diarrhea, fatigued or tired, nausea/ vomiting, pain, and trouble eating/swallowing). Univariate analyses were used to identify factors that were individually associated with H-MST scores. These factors were included in a multivariable model; backwards elimination identified factors independently associated with H-MST scores. A significance level of $p<0.05$ was used for model selection including both univariate analyses and multivariable analyses with backwards elimination. All data processing and statistical analyses were performed utilizing the SAS version 9.4 software application.

\section{Results}

\section{Patient characteristics}

A total of 7479 unique subjects with either clinical or EDS data were retrieved (Fig. 1). Of these, complete case records as defined above were available for 4272. Six hundred and eighty-seven were removed due to "unknown" or "not stated" BMI, race, sex, or stage in the tumor registry with a final dataset of 3585 subjects for analysis. The MST completed closest to date of cancer diagnosis was analyzed; median time from diagnosis to MST was 20 days (25-75 IQR, 11-33 days). Median age was 64 (25-75 IQR, 55-72) with range from 18 to 98 . Most had stages I-III disease $(80 \%): 33 \%=$ stage I, $27 \%=$ stage II, and $20 \%=$ stage III. Median weight in pounds was 175 (25-75 IQR, 148-207) range 70-514. Additional clinical and demographic data are in Table 2.

\section{Malnutrition screening tool scores}

The raw MST scores for all are in Supplementary Table S1. High MST (H-MST) as defined as score of $\geq 2$ to 5 was present in 1002 (28\%) of which 2\% scored 5, 4\% scored 4 , and $22 \%$ scored 2-3. H-MST versus L-MST scores by demographics and symptom burden are in Table 2. Figure 2 provides H-MST prevalence by cancer diagnostic group. As shown, H-MST was present in 58\% of upper GI cancers, $42 \%$ in both lower GI cancers and thoracic cancers, and 34\% of head and neck cancer, and $10 \%$ of breast cancers.

\section{Multivariable analysis of associations with high MST}

Univariable logistic regression for H-MST identified age $\geq 65$, Black race, cancer diagnostic group, male sex, stage IV disease, BMI, and higher symptom scores to be associated with a greater probability of H-MST (all $p<0.001$ ). As shown in Table 3 , multivariable regression confirmed Black race, cancer diagnostic group, low BMI, and stage IV disease to be independently associated with H-MST. Additionally, higher scores for depression, distress, fatigue, and trouble eating/swallowing were all 
Fig. 1 Patient flow. Abbreviations: $\mathrm{N}=$ number; $\mathrm{EDS}=$ electronic distress screening; $\mathrm{BMI}=$ body mass index

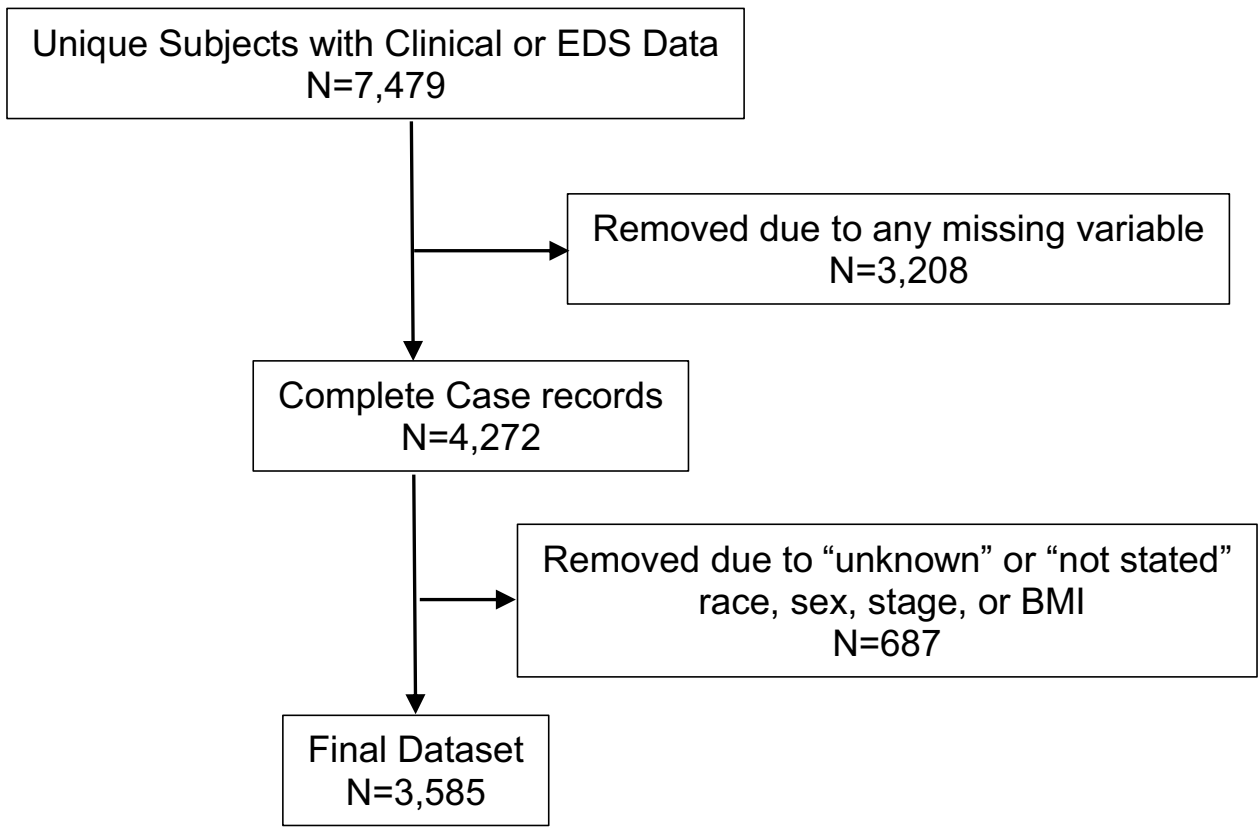

independently associated with H-MST, whereas anxiety, constipation/diarrhea, nausea/vomiting, and pain were not.

\section{Discussion}

In this US cohort of over 3000 ambulatory solid tumor patients, over one-quarter were at high risk for malnutrition at diagnosis. There was significant variation with the greatest risk of malnutrition in those with lower GI, upper GI, and thoracic cancers, advanced stage disease, Black race, and higher symptom scores.

These striking results are consistent with oncology cohort studies, particularly those that included early-stage disease and breast cancer. [29-31] Despite nearly one-third being at high risk of malnutrition, our observed rate is lower than other studies which suggests variation in prevalence is driven by the specific oncology population. For example, in the prospective Italian PreMiO study, of nearly 2000 ambulatory solid and hematologic cancer patients, 51\% had malnutrition based on the mini nutritional assessment, a validated measure of nutritional status in the elderly. [7] This study, unlike the current analysis, included more GI cancers and higher rates of stage IV disease, both factors associated with higher rates of malnutrition.

Beyond the cancer site, the highest risk of malnutrition was observed in those with a low BMI. However, a notable observation was the high rates of malnutrition risk in those with high BMI. Of those classified as overweight or obese by BMI, H-MST was observed in $24 \%$ and $20 \%$, respectively. The obesity epidemic means $40-60 \%$ of all new cancer diagnoses present in those with obesity, and this new clinical picture likely reduces the identification of malnutrition by practicing clinicians. A portion of these malnourished obese patients will have sarcopenia, which is associated with poor oncologic outcomes. [32, 33] Although the MST does not directly identify sarcopenia, such screening tools might identify at-risk patients for referral to nutritional services for further evaluation. This highlights the importance of malnutrition screening in all cancer patients independent of weight or BMI at diagnosis. Future work should evaluate which highrisk groups might benefit from the addition of tools used to screen for sarcopenia, such as the SARC-F. [34] However, like the MST, any such positive screening assessment should prompt a comprehensive nutrition assessment and confirm malnutrition diagnosis and appropriate interventions. [35]

Unique to this analysis and related to our catchment area, Black patients accounted for one-fifth of those studied. They had numerically higher H-MST rates than White or other races, and this was independently associated with a nearly twofold increase in high malnutrition risk. Smaller US cancer studies using varied screening tools have not reported the prevalence of malnutrition by race. [31,36] Notably, Black race is correlated with higher malnutrition rates in community-dwelling older adults in non-cancer populations. [37] Sociodemographic factors, not included in the current analysis, disproportionately affect racial minorities and might also account for our findings and should be explored further in future investigations. The demographics analyzed suggest generalizability of these findings to other diverse US communities.

Aging, changes in end-organ function, and body composition remain well-documented risk factors for malnutrition in non-cancer and cancer illnesses. [22, 38, 39] In a study of patients with varying cancer diagnoses in which half were 
Table 2 High versus low MST scores by demographics and patientreported symptoms

\begin{tabular}{|c|c|c|c|}
\hline Variable & High MST & Low MST & Total \\
\hline & $n(\%)^{\mathrm{a}}$ & $n(\%)^{\mathrm{a}}$ & $n(\%)^{\mathrm{b}}$ \\
\hline All & $1002(28)$ & $2583(72)$ & $3585(100)$ \\
\hline \multicolumn{4}{|l|}{ Raw MST score } \\
\hline 0 & & 2109 & $2109(59)$ \\
\hline 1 & & 474 & $474(13)$ \\
\hline 2 & 492 & & $492(14)$ \\
\hline 3 & 301 & & $301(8)$ \\
\hline 4 & 128 & & $128(4)$ \\
\hline 5 & 81 & & $81(2)$ \\
\hline \multicolumn{4}{|l|}{ Age group } \\
\hline$\leq 50$ & $132(21)$ & $506(79)$ & $638(18)$ \\
\hline$>50$ to $<65$ & $373(30)$ & $892(70)$ & $1265(35)$ \\
\hline$\geq 65$ & $497(30)$ & $1185(70)$ & $1682(47)$ \\
\hline \multicolumn{4}{|l|}{ Gender } \\
\hline Female & $524(24)$ & $1683(76)$ & $2207(62)$ \\
\hline Male & $478(35)$ & $900(65)$ & $1378(38)$ \\
\hline \multicolumn{4}{|l|}{ Race } \\
\hline White & $766(26)$ & $2137(74)$ & $2903(81)$ \\
\hline Black & $213(35)$ & $391(65)$ & $604(17)$ \\
\hline Other & $23(30)$ & $55(70)$ & $78(2)$ \\
\hline \multicolumn{4}{|l|}{ Stage } \\
\hline I-III & $650(23)$ & $2222(77)$ & $2872(80)$ \\
\hline IV & $352(49)$ & $361(51)$ & $713(20)$ \\
\hline \multicolumn{4}{|l|}{ Median BMI } \\
\hline$<18.5$ & $70(67)$ & $34(33)$ & $104(3)$ \\
\hline$\geq 18.5$ to $<25$ & 404 (38) & $653(62)$ & $1057(29)$ \\
\hline$\geq 25$ to $<30$ & $271(24)$ & $841(76)$ & $1112(31)$ \\
\hline$\geq 30$ & $257(20)$ & $1055(80)$ & $1312(37)$ \\
\hline \multicolumn{4}{|l|}{ Alcohol or tobacco use } \\
\hline Yes & $755(30)$ & $1772(70)$ & $2527(71)$ \\
\hline No & 247 (23) & $811(77)$ & $1058(29)$ \\
\hline \multicolumn{4}{|l|}{ Anxiety per GAD-2 } \\
\hline Yes & $328(41)$ & $480(59)$ & $808(22)$ \\
\hline No & $674(24)$ & $2103(76)$ & $2777(78)$ \\
\hline \multicolumn{4}{|l|}{ Depression per PHQ-2 } \\
\hline Yes & $313(49)$ & $328(51)$ & $641(18)$ \\
\hline No & $689(23)$ & $2255(77)$ & $2944(82)$ \\
\hline \multicolumn{4}{|l|}{ Distress per $0-10$ scale } \\
\hline $\operatorname{High}(\geq 4 / 10)$ & $777(33)$ & $1568(67)$ & $2527(65)$ \\
\hline Low & $225(18)$ & $1015(82)$ & $1240(35)$ \\
\hline \multicolumn{4}{|c|}{ Symptoms per 0-10 scale, median (25-75 IQR) } \\
\hline Pain & $5(2-7)$ & $2(0-5)$ & $2(0-5)$ \\
\hline Fatigue & $6(4-8)$ & $3(1-6)$ & $5(2-7)$ \\
\hline Nausea/vomiting & $0(0-3)$ & $0(0-0)$ & $0(0-0)$ \\
\hline Diarrhea/constipation & $2(0-5)$ & $0(0-2)$ & $0(0-4)$ \\
\hline Trouble eating/swallowing & $3(0-6)$ & $0(0-0)$ & $0(0-2)$ \\
\hline
\end{tabular}

Abbreviations: $M S T$ malnutrition score tool; $N$ number; $B M I$ body mass index; IQR interquartile range; $P H Q-2$ Patient Health Questionnaire 2-item; $G A D-2$ Generalized Anxiety Disorder 2-item

${ }^{\text {a }}$ Row percentage

${ }^{\mathrm{b}}$ Column percentage outpatients, $90 \%$ had at least one nutrition barrier when referred to a dietitian. [40] It is noteworthy that loss of appetite, nausea, and early satiety were the most common nutrition barriers. The number of nutrition barriers was significantly associated with reduced food intake and greater weight loss. In the elderly, cognitive issues, decreased mobility, frailty, poor dentition, polypharmacy, and social isolation can also contribute to malnutrition. [41] In our sample, age was not independently associated with H-MST in multivariable analysis. However, multiple symptoms were independently associated with H-MST, including depression, distress, fatigue, and trouble eating/swallowing. Studies of symptoms have observed their presence to be associated with a greater risk of malnutrition, which appears to rise with age. [42] Therefore, when feasible, a comprehensive geriatric assessment should be performed to identify additional contributing factors to malnutrition in older adults with cancer.

Although this study identified malnutrition risk in a large US cancer center, certain limitations are noteworthy. As a goal was to evaluate the prevalence of malnutrition risk by clinicodemographic variables, we limited the analysis to persons with complete case records. A sensitivity analysis found similar MST distribution with 27\% H-MST and comparable age, race, gender, and cancer diagnostic groupings between the final dataset and all subjects (Supplementary Table S1). Therefore, our complete case analysis is likely representative of the larger cohort. Additionally, this was a cross-sectional analysis and included MST scores only at diagnosis. Third, our analysis was based only on patient self-reported MST. Due to limitations of the institutional database, we were unable to analyze the impact of malnutrition on short-term and long-term oncologic outcomes like treatment tolerability and survival. Lastly, future studies should control for additional comorbidities to minimize residual confounding.

Malnutrition screening remains uncommon in routine clinical care. [43, 44] Given the mandate to screen for psychological distress by the American College of Surgeons and Commission on Cancer, electronic screening has been shown to be feasible in large cancer centers. [45] As we have done, incorporating malnutrition screening within such established electronic screening tools might increase early identification. [43] Using MST as a brief, rapidly administered tool would minimize time commitments by health care professionals. Further research is needed to optimize the role of electronic screening tools and its integration into clinical care.

The high risk of malnutrition in this cohort, beyond those with low BMI at presentation, supports routine malnutrition screening at diagnosis. Future studies should evaluate ongoing screening throughout the cancer trajectory. Understanding such findings will inform future interventional studies targeting malnutrition early, cost-benefit analyses, registered dietitian nutritionist staffing patterns, and health outcomes in oncology. 


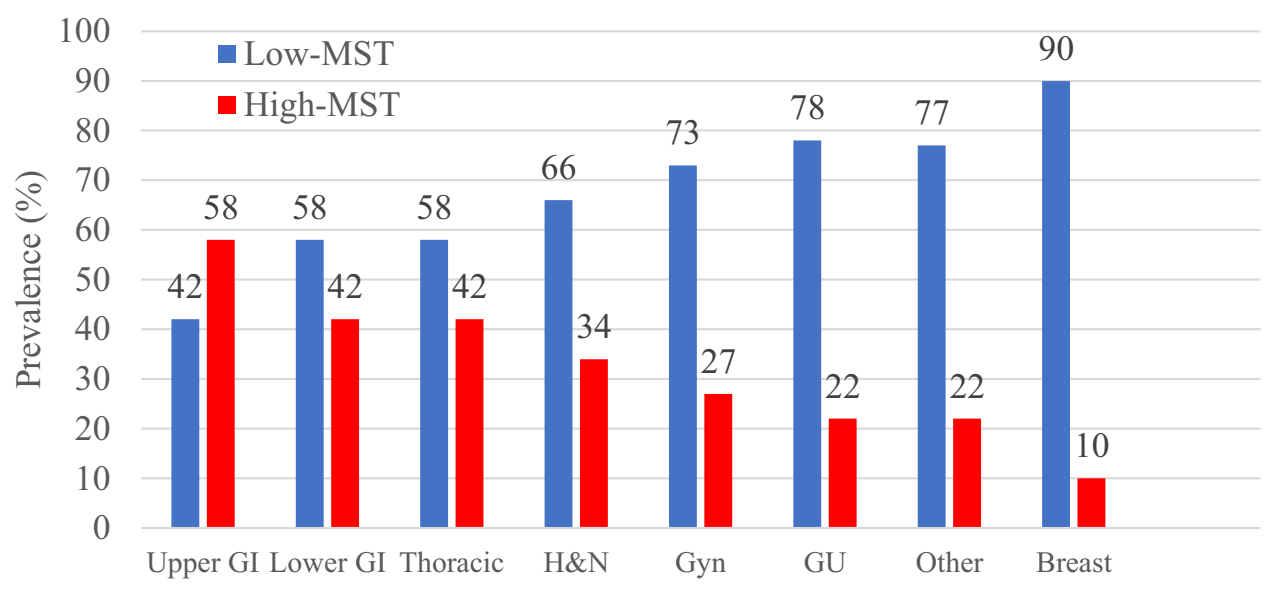

Cancer Diagnostic Group ${ }^{1}$

Fig. 2 Prevalence of low versus high MST by cancer diagnostic group. ${ }^{1}$ Cancer diagnostic groups included upper GI: any biliary and liver $(n=108)$, esophagus $(n=58)$, gastric $(n=60)$, pancreas $(n=157)$; lower GI: anal $(n=18)$, colon $(n=228)$, rectum $(n=77)$, small bowel $(n=16)$; thoracic: bronchus and lung $(n=457)$; H\&N: any head and neck primary $(n=136)$; Gyn: cervix $(n=60)$, other female genital $(n=28)$, ovarian $(n=60)$, uterine $(n=170)$, vagina and vulva $(n=18)$; GU: prostate $(n=180)$, renal cell carcinoma $(n=131)$, testis $(n=9)$, urinary bladder and ureter $(n=113)$; other: malignant melanoma $(n=199)$, cancer of unknown primary $(n=193)$, sarcoma $(n=50)$, central nervous system $(n=3)$, thyroid $(n=40)$; breast: any mammary primary $(n=1016)$. Abbreviations: MST $=$ malnutrition score tool; $\mathrm{N}=$ number; upper $\mathrm{GI}=$ upper gastrointestinal; lower $\mathrm{GI}=$ lower gastrointestinal; GYN= gynecologic; $\mathrm{GU}=$ genitourinary; $\mathrm{H} \& \mathrm{~N}=$ head and neck
Table 3 Multivariable logistic regression of associations with high MST by demographics and symptom burden

\begin{tabular}{|c|c|c|c|c|}
\hline \multirow[b]{2}{*}{ Factor } & \multirow[b]{2}{*}{ Reference level } & \multirow[b]{2}{*}{ Factor level } & \multicolumn{2}{|c|}{ Multivariable results } \\
\hline & & & $\mathrm{OR}(\mathrm{CI})$ & P-value \\
\hline \multirow[t]{3}{*}{ Race } & & & & $<0.001$ \\
\hline & White & Black & $1.91(1.52,2.39)$ & \\
\hline & & Other & $1.26(0.69,2.29)$ & \\
\hline \multirow[t]{8}{*}{ Diagnosis } & & & & $<0.001$ \\
\hline & Breast & Upper GI & $5.72(4.12,7.93)$ & \\
\hline & & Lower GI & $4.69(3.39,6.52)$ & \\
\hline & & Thoracic & $3.25(2.38,4.44)$ & \\
\hline & & GYN & $2.59(1.83,3.67)$ & \\
\hline & & GU & $2.08(1.48,2.92)$ & \\
\hline & & Other & $2.03(1.46,2.82)$ & \\
\hline & & $\mathrm{H} \& \mathrm{~N}$ & $1.63(1.00,2.65)$ & \\
\hline Stage & I-III & IV & $1.76(1.43,2.16)$ & $<0.001$ \\
\hline \multirow[t]{4}{*}{ BMI } & & & & $<0.001$ \\
\hline & $\geq 30$ & $<18.5$ & $4.16(2.50,6.91)$ & \\
\hline & & $\geq 18.5,<25$ & $2.13(1.71,2.65)$ & \\
\hline & & $\geq 25,<30$ & $1.19(0.95,1.49)$ & \\
\hline Distress & No & Yes & $1.25(1.01,1.54)$ & 0.040 \\
\hline Depression & No & Yes & $1.33(1.06,1.67)$ & 0.014 \\
\hline Fatigue & Continuous & - & $1.15(1.11,1.19)$ & $<0.001$ \\
\hline $\begin{array}{l}\text { Trouble eating or swal- } \\
\text { lowing }\end{array}$ & Continuous & - & $1.22(1.18,1.26)$ & $<0.001$ \\
\hline
\end{tabular}

Abbreviations: $M S T$ malnutrition score tool; $N$ number; $O R$ odds ratio; $C I$ confidence interval; $G I$ gastrointestinal; $G Y N$ gynecologic; $G U$ genitourinary; $H \& N$ head and neck; $B M I$ body mass index; $E t O H$ alcohol 


\section{Conclusions}

Over one-quarter of solid tumor oncology outpatients at diagnosis who are screened are at high risk of malnutrition. There was significant variation in malnutrition risk by cancer site, stage, race, and greater symptom burden. This is the largest study of malnutrition screening within a US ambulatory oncology population. These findings support current efforts to standardize malnutrition screening to optimally target nutritional resource allocation to the highest risk groups early in their cancer trajectories.

Supplementary Information The online version contains supplementary material available at https://doi.org/10.1007/s00520-021-06612-z.

Author contribution Kunal C. Kadakia: conceptualization, data curation, formal analysis, investigation, methodology, validation, visualization, writing — original draft, and writing — review and editing.

James T. Symanowski: data curation, formal analysis, methodology, validation, and writing-review and editing.

Aynur Aktas: conceptualization, writing - original draft, and writing-review and editing.

Michele L. Szafranski: conceptualization, writing-original draft, and writing-review and editing.

Jonathan C. Salo: writing—original draft, and writing—review and editing.

Patrick L. Meadors: conceptualization, methodology, writingoriginal draft, and writing - review and editing.

Declan Walsh: conceptualization, methodology, writing-original draft, and writing-review and editing.

\section{Data availability NA.}

Code availability NA.

\section{Declarations}

Ethics approval All procedures performed in studies involving human participants were in accordance with the ethical standards of the institutional and/or national research committee and with the 1964 Helsinki declaration and its later amendments or comparable ethical standards.

Consent to participate NA. Informed consent was not obtained as this was a retrospective study.

\section{Consent for publication NA.}

Competing interests The authors declare no competing interests.

\section{References}

1. Baracos VE, Martin L, Korc M et al. Cancer-associated cachexia. Nat Publ Gr 2018;4. https://doi.org/10.1038/nrdp. 2017.105.

2. Sorensen J, Kondrup J, Prokopowicz J et al (2008) EuroOOPS: an international, multicentre study to implement nutritional risk screening and evaluate clinical outcome. Clin Nutr 27:340-349
3. Amaral TF, Antunes A, Cabral S et al (2008) An evaluation of three nutritional screening tools in a Portuguese oncology centre. J Hum Nutr Diet 21:575-583

4. Bozzetti F, Mariani L, Lo Vullo S et al (2012) The nutritional risk in oncology: a study of 1,453 cancer outpatients. Support Care Cancer 20:1919-1928

5. Isenring E, Cross G, Kellett E et al (2010) Nutritional status and information needs of medical oncology patients receiving treatment at an Australian public hospital. Nutr Cancer 62:220-228

6. Sharma D, Kannan R, Tapkire R et al (2015) Evaluation of nutritional status of cancer patients during treatment by patient-generated subjective global assessment: a hospital-based study. Asian Pac J Cancer Prev 16:8173-8176

7. Muscaritoli M, Lucia S, Farcomeni A et al (2017) Prevalence of malnutrition in patients at first medical oncology visit: the PreMiO study. Oncotarget 8:79884-79896

8. Rhondali W, Chisholm GB, Daneshmand M et al (2013) Association between body image dissatisfaction and weight loss among patients with advanced cancer and their caregivers: a preliminary report. J Pain Symptom Manage 45:1039-1049

9. Oberholzer R, Hopkinson JB, Baumann K et al (2013) Psychosocial effects of cancer cachexia: a systematic literature search and qualitative analysis. J Pain Symptom Manage 46:77-95

10. Monteiro-Grillo I, Vidal PM, Camilo ME et al (2004) Cancer: disease and nutrition are key determinants of patients? quality of life. Support Care Cancer 12:246-252

11. Martin L, Senesse P, Gioulbasanis I et al (2015) Diagnostic criteria for the classification of cancer-associated weight loss. J Clin Oncol 33:90-99

12. Orrevall Y, Tishelman C, Permert J et al (2009) Nutritional support and risk status among cancer patients in palliative home care services. Support Care Cancer 17:153-161

13. Huhmann MB, Cunningham RS (2005) Importance of nutritional screening in treatment of cancer-related weight loss. Lancet Oncol 6:334-343

14. Isenring EA, Capra S, Bauer JD (2004) Nutrition intervention is beneficial in oncology outpatients receiving radiotherapy to the gastrointestinal or head and neck area. Br J Cancer 91:447

15. Ravasco P, Monteiro-Grillo I, Vidal PM et al (2005) Dietary counseling improves patient outcomes: a prospective, randomized, controlled trial in colorectal cancer patients undergoing radiotherapy. J Clin Oncol 23:1431-1438

16. Arends J, Baracos V, Bertz H et al (2017) ESPEN expert group recommendations for action against cancer-related malnutrition. Clin Nutr 36:1187-1196

17. Castillo-Martinez L, Castro-Eguiluz D, Copca-Mendoza ET et al (2018) Nutritional assessment tools for the identification of malnutrition and nutritional risk associated with cancer treatment. Rev Investig Clin 70:121-125

18. Leuenberger M, Kurmann S, Stanga Z (2010) Nutritional screening tools in daily clinical practice: the focus on cancer. Support Care Cancer 18:17-27

19. Ferguson ML, Bauer J, Gallagher B et al (1999) Validation of a malnutrition screening tool for patients receiving radiotherapy. Australas Radiol 43:325-327

20. Ferguson M, Capra S, Bauer J et al (1999) Development of a valid and reliable malnutrition screening tool for adult acute hospital patients. Nutrition 15:458-464

21. Isenring E, Cross G, Daniels L et al (2006) Validity of the malnutrition screening tool as an effective predictor of nutritional risk in oncology outpatients receiving chemotherapy. Support Care Cancer 14:1152-1156

22. de Pinho NB, Martucci RB, Rodrigues VD et al (2020) High prevalence of malnutrition and nutrition impact symptoms in older patients with cancer: results of a Brazilian multicenter study. Cancer 126:156-164 
23. von Elm E, Altman DG, Egger M et al (2014) The strengthening the reporting of observational studies in epidemiology (STROBE) statement: guidelines for reporting observational studies. Int J Surg 12:1495-1499

24. Obesity: preventing and managing the global epidemic. Report of a WHO consultation - PubMed. Available at https://pubmed.ncbi. nlm.nih.gov/11234459/. Accessed February 25, 2021.

25. Kroenke K, Spitzer RL, Williams JBW et al (2007) Anxiety disorders in primary care: prevalence, impairment, comorbidity, and detection. Ann Intern Med 146:317-325

26. Kroenke K, Spitzer RL, Williams JBW (2003) The patient health questionnaire-2: Validity of a two-item depression screener. Med Care 41:1284-1292

27. Wagner LI, Pugh SL, Small W et al (2017) Screening for depression in cancer patients receiving radiotherapy: feasibility and identification of effective tools in the NRG Oncology RTOG 0841 trial. Cancer 123:485-493

28. Riba MB, $\theta$ C, Donovan KA et al. NCCN guidelines version 2. 2020 Distress Management Continue NCCN Guidelines Panel Disclosures. , 2020.

29. Schneider CK, Bressler T (2020) Malnutrition screening: an interprofessional approach in outpatient oncology. Clin J Oncol Nurs 24:E28-E33

30. Hauner H, Kocsis A, Jaeckel B et al (2020) Prevalence of malnutrition risk in patients of cancer outpatient clinics - a crosssectional survey. Dtsch Medizinische Wochenschrift 145:E1-E9

31. De Groot LM, Lee G, Ackerie A et al (2020) Malnutrition screening and assessment in the cancer care ambulatory setting: mortality predictability and validity of the patient-generated subjective global assessment short form (PG-SGA SF) and the GLIM criteria. Nutrients 12:1-13

32. Prado CM, Lieffers JR, McCargar LJ et al (2008) Prevalence and clinical implications of sarcopenic obesity in patients with solid tumours of the respiratory and gastrointestinal tracts: a population-based study. Lancet Oncol 9:629-635

33. Huiskamp LFJ, Chargi N, Devriese LA et al (2020) The predictive value of low skeletal muscle mass assessed on cross-sectional imaging for anti-cancer drug toxicity: a systematic review and meta-analysis. J Clin Med 9:3780

34. Grant W, Al-Obaidi M, Chen D et al (2021) SARC-F for screening of sarcopenia among older adults with cancer. Cancer 127:1469-1475
35. Malas FÜ, Kara M, Özçakar L (2021) SARC-F to screen or diagnose sarcopenia in cancer? A point-blank refusal. Cancer 127:2158-2158

36. Zhang X, Pang L, Sharma SV et al (2019) Prevalence and factors associated with malnutrition in older patients with cancer. $\mathrm{J}$ Geriatr Oncol 10:763-769

37. Sadarangani T, Missaelides L, Brody A et al. Racial disparities in nutritional risk and its association with chronic disease and health outcomes among community-dwelling older adults in the adult day health setting (P04-121-19). Curr Dev Nutr 2019;3. https:// doi.org/10.1093/cdn/nzz051.p04-121-19.

38. Abd Allah ES, Gad HMM, Abdel-Aziz HR (2020) Nutritional status and its contributing factors among older adults with cancer receiving chemotherapy. Clin Nurs Res 29:650-658

39. Zhang X, Pang L, Sharma SV et al (2020) Malnutrition and overall survival in older patients with cancer. Clin Nutr. https://doi.org/ 10.1016/j.clnu.2020.06.026

40. Lorton CM, Griffin O, Higgins K et al (2020) Late referral of cancer patients with malnutrition to dietitians: a prospective study of clinical practice. Support Care Cancer 28:2351-2360

41. X Z, BJ E. Malnutrition in older adults with cancer. Curr Oncol Rep 2019;21. https://doi.org/10.1007/S11912-019-0829-8.

42. de Pinho NB, Martucci RB, Rodrigues VD et al (2019) Malnutrition associated with nutrition impact symptoms and localization of the disease: results of a multicentric research on oncological nutrition. Clin Nutr 38:1274-1279

43. Walsh D, Szafranski M, Aktas A et al. Malnutrition in cancer care: time to address the elephant in the room. J Oncol Pract 2019;15. https://doi.org/10.1200/JOP.19.00165.

44. Trujillo EB, Claghorn K, Dixon SW et al. Inadequate nutrition coverage in outpatient cancer centers: results of a national survey. J Oncol 2019;2019. https://doi.org/10.1155/2019/7462940.

45. JW N, M R, K B et al. Distress screening through patient-reported outcomes measurement information system (PROMIS) at an academic cancer center and network site: implementation of a hybrid model. JCO Oncol Pract 2021;:OP.20.00473.

Publisher's note Springer Nature remains neutral with regard to jurisdictional claims in published maps and institutional affiliations. 